\title{
Immunological aspects of chronic venous disease pathogenesis
}

\author{
EWA GRUDZINSSKA, ZENON PAWEŁ CZUBA
}

Department of Microbiology and Immunology in Zabrze, Medical University of Silesia, Katowice, Poland

\begin{abstract}
Chronic venous disease (CVD) is a very common health problem concerning up to $1 / 3$ of the society. Although venous hypertension and valvular incompetence have been long known to be crucial for development of the illness, its exact aetiology remains unclear. Recent findings indicate that inflammatory processes may be crucial for development of incompetent valves and vein wall remodelling. One of the most interesting theories describes "leucocyte trapping" as the mechanism responsible for elevated vein wall permeability and oxidative stress in the veins. At the same time, the cytokine profile of the blood in incompetent veins has not been thoroughly examined. Popular anti-inflammatory drugs relieve some symptoms but do not have much proved effects in prevention and treatment.

We intend to summarize the existing knowledge of the immunological aspects of CVD in order to emphasize its importance for understanding the aetiology of this illness. We also wish to indicate some aspects that remain to be studied in more detail.
\end{abstract}

Key words: cytokines, chronic venous disease, varices, veins, leukocyte trapping.

(Centr Eur J Immunol 2014; 39 (4): 525-531)

\section{Introduction}

The lower extremity superficial veins (great saphenous vein - GSV and small saphenous vein - SSV) are responsible for draining the skin and subcutaneous tissue. They are connected with the deep vein system by perforators. All these veins are equipped with valves responsible for preventing the blood reflux. If any derangement of this system occurs, chronic vein incompetence sets in. Blood stasis causes symptoms like leg heaviness, oedema, pigmentation and ulceration and the most severe complication is thrombosis which may lead to pulmonary thromboembolism and death.

Chronic venous disease (CVD) is a very common problem, especially in Western countries, in industrial regions [1]. According to numerous papers, varices may concern as much as $1 / 5-1 / 3$ of the population with a slight predominance of women [2-7]. The risk factors are family history [8], age, obesity [9], female gender and pregnancy $[1,4]$. The hereditary factors and age seem to be the most important factors promoting the illness $[3,4,10]$.

Aetiology of venous insufficiency remains obscure. Valvular insufficiency and reflux have been long considered crucial for formation of varices and indeed, valves of varicose veins are widened and hypotrophic and the vessel lumen is larger than in healthy veins [11]. Nevertheless, the primary reason for these changes remains unclear. Recent publications suggest that inflammation may be the fundamental element in the pathogenesis of venous insufficiency $[12,13]$, and the phenomenon of the "leucocyte trap" is of great interest [14-18].

The following review aims at summarizing the information on immunological processes which could be of importance for the venous disease pathogenesis. We included only a few of the publications on venous ulcers because immunological changes in this stage of venous insufficiency may be largely dependent on bacterial presence (including endogenous microflora and pathogenic microflora like Staphylococcus aureus and Pseudomonas aeruginosa) [19], therefore they may differ a lot from the immunological state underlying the disease.

\section{Leukocytes involved in chronic venous disease}

Multiple works point to the possibility of macrophage-released growth factors, proinflammatory cytokines, matrix metalloproteinases (MMP) and adhesion molecules being involved in the aetiology of varicose veins $[15,16]$. Out of all white blood cells it is the macrophage/monocyte group which is represented in greatest numbers in incompetent veins of lower extremities. These cells are located differently than in healthy veins, i.e. in proximity of valves and vein walls $[15,16,20]$. They are present in adventitia and in vasa vasorum but not in the muscular layer [21].

Correspondence: Zenon Paweł Czuba, PhD, DSc, Department of Microbiology and Immunology in Zabrze, Medical University of Silesia, H. Jordana 19, 41-808 Zabrze, Poland, e-mail: zczuba@sum.edu.pl 
Macrophage infiltration is most intensive near the valves and through proteolytic enzymes it may contribute to their destruction and mechanical weakening [20]. Patients suffering from venous ulcers have a greater number of monocyte-platelet complexes, both in lower and in upper extremities [22]. The number of these complexes does not diminish even after radical surgery of GSV, which indicates that they must be present before venous hypertension evolves and so they take part in early pathogenesis [23].

Most publications claim that the number of $\mathrm{T}$ lymphocytes in varices is the same as in healthy veins [15] and there are very few papers on their role in aetiology of venous insufficiency. Bujan et al. [24] observed an increase in CD4+ cells in the varicose vein tissue. The localization of these cells was different depending on age: in the older group (over 50 years of age) they infiltrated subendothelium and valves, while in the younger group they were found in the adventitial and medial layers. Lymphocytes $\mathrm{B}$ were also found in greater numbers in varices than in healthy specimens, however, this was true only in the older group of patients.

We would also like to mention here some studies of arterial diseases, which point to a significant role of T-cells in pathogenesis even though, just like in venous disease, their number is the same as in healthy patients. In arteriosclerosis the macrophage: lymphocyte T ratio is about $4: 1$. However, it is not the number, but the activity of T-cells that proves important. In atherogenesis mostly Th1 and interferon $\gamma$ (IFN- $\gamma$ ), as well as interleukin (IL)-1, IL-12 and IL-18 promote the illness [25]. Th17 lymphocytes are still a novelty; their role in atherogenesis (and even more so in venous insufficiency) is unclear. In atherosclerotic plaques small amounts of IL-17 mRNA were found, but IL-17 protein was abundant $[25,26]$. Ju et al. implicate that IL-17A has a large role in aortic aneurysm formation in mice [27]. These studies show that lack of significant differences in lymphocyte number in patients in comparison with healthy people does not necessarily mean that these cells have no impact on illness pathogenesis. Lymphocyte activity in venous illness may be an interesting target for future studies, just like it is in atherogenesis.

Neutrophil population in wall and in blood of varicose veins is very small $[15,28]$. In one study flow cytometry showed lack of neutrophil activation in varices, regardless of ulceration [29]. There were no differences in neutrophil-platelet aggregation as well [22], although in the presence of elevated shear force adhesion between these cells was increased and followed by aggregation via P-selectin and its ligand, glycoprotein PSGL-1 [30].

It is postulated that neutrophils have an important role in venous insufficiency pathogenesis. Their absence in venous blood is explained by "trapping" in tiny vessels [17, 28]. The "leukocyte trap" mechanism is described as infiltration of activated neutrophils caused by hypertension, hypoxia and stasis [17, 28, 31, 32].
In normal environment neutrophils do not contact each other and their halftime is about $7 \mathrm{~h}$ [33]. Inflammation lengthens this time and causes neutrophil aggregation and their adhesion to endothelium [33, 34]. First they adhere to endothelium via $\mathrm{P}, \mathrm{E}$ and $\mathrm{L}$ selectin and they roll along the vessel wall [35]. Next, the CD11/CD18 complex along with endothelial intercellular adhesion molecules ICAM1 and ICAM-2 cause strong adhesion [36, 37]. As a consequence neutrophils produce free radicals and proteases [35]. The adhesion process may be initiated by IL-8, PAF (platelet activation factor), active complement complex, arachidonic acid metabolites and other cytokines like IFN- $\gamma$ $[34,35,38]$. Tumor necrosis factor $\alpha(\mathrm{TNF}-\alpha)$ increases neutrophil activation (adhesion, degranulation and free radical creation) via p55 and p7 [39].

In accordance with the "leucocyte trap" theory it has been shown that indeed, more neutrophils adhere to endothelium in veins incubated in hypoxic conditions [32, 33]. What is more, neutrophils adhering to the endothelium are active, with elevated cytoplasm calcium levels and considerable NO and LTB4 production [34, 38, 40]. Apart from hypoxia, also venous hypertension in standing position is related to inflammatory reaction [41], elevated L-selectin plasma levels and its decrease in neutrophils and monocytes [17]. Adhesion of active neutrophils and monocytes decreases after resting [17, 31]. Venous stasis causes release of IL-1b, IL-6 and TNF- $\alpha$ as well [16], suggesting endothelial activation $[16,41]$. It is suggested that in plasma of varicose veins an unknown neutrophil activating factor is present because neutrophils from healthy patients added to plasma of patients suffering from varicose veins become activated and create multiple pseudopodia [28].

\section{Pathological factors of vein wall remodelling}

The role of free radicals in venous disease is well described. Enhanced production of free radicals by neutrophils in varicose veins was noted in numerous publications [14, 18, 42-44]. It seems that this production is performed mostly by NADPH oxidase (NOX) and by nitric oxide synthase (NOS) [43, 45], and in thrombophlebitis the xanthine oxidase is also relevant [14]. At the same time antioxidative mechanisms are weakened - superoxide dismutase (SOD) has lowered activity and the total antioxidative potential measured by the FRAP test (Ferric Reducing Ability of Plasma test) is small [46]. Free radicals, especially reactive forms of oxygen and nitrogen produced by active neutrophils and macrophages, cause degradation of the main protein components of the venous wall - collagen and elastin. Elevated concentrations of lipid peroxidation products like malonyldialdehyde (MDA), myeloperoxidase (MPO) and reactive thiobarbituric acid derivatives (TBARs) which are found in varices indicate elevated lipid peroxidation which destroys cell membrane, setting 
free additional proteases $[14,46]$. Moreover, it has been shown that incompetent venous fragments contain more ferric ions in comparison to normal vein fragments, there is also a greater concentration of zinc and copper which are SOD cofactors [47]. In incompetent vein fragments more oxidative damage and TBARs were found. Authors of this study emphasize the role of iron in oxidative stress and in chronic inflammation [47]. Removal of the incompetent vein causes a prominent decrease in oxidative stress markers in venous blood [48].

Endothelial protein degradation is also caused by elevated activity and expression of MMPs connected to hypertension and stasis $[13,41,49,50]$. These damages in turn cause leukocyte infiltration and activation, manifested by elastase and lactoferrin increase [51, 52]. Elastase content is elevated in plasma of varicose veins regardless of inflammatory complications (like lipodermatosclerosis or ulceration) [52]. For venous ulcers, MMP-2 and MMP-9 appear as crucial factors which impair healing and the MMP-9 levels are elevated in wound fluid as well as in plasma $[50,53]$. As the chronic wounds heal, a decrease in MMP-2 and MMP-9 is noted [54].

Gomez et al. showed decreased levels of active MMP-1 and MMP-2 in varices with increase of tissue inhibitors of metalloproteinases (TIMP-1 and TIMP-2) [55]. Sansilvestri, however, noted increased production of MMP-1, -2 and -3 with no difference in MMP-7 and -9 or TIMP-1, -2 and -3 [56]. Decrease in mitochondrial prostaglandin $\mathrm{E}_{2}$ and its receptor, EP4 accompanied by increase in 15-hydroxyprostaglandin dehydrogenase, which is its only degrading enzyme, were also found. The authors conclude that the observed changes take part in thickening of the venous wall and suggest that these changes may have a protective impact, strengthening the vein during blood stasis [55].

The deranged activity of leukocytes along with factors released from platelets and macrophages leads to proinflammatory activity of endothelium and it is considered a potential source of pathologic vein wall remodelling, vein lumen widening and valve incompetency with following varices formation $[31,57]$.

At the same time there is no correlation between the intensity of systemic inflammatory reaction and the symptoms noted by the patients [58].

A rat model of venous hypertension (arterio-venous fistula) showed that the isolated increase of pressure does not cause reflux: the venous diameter grows but the valves remain competent. Only after some time, with increasing migration of monocytes/macrophages and lymphocytes $\mathrm{T}$ to the valves (and elevated expression of selectin $P$ and ICAM-1 on endothelial cells) venous insufficiency sets in. Authors of this study administered a flavonoid prior to creating the fistula which to some extent decreased expression of adhesins and lymphocyte $\mathrm{T}$ infiltration [59].

As a result of inflammatory changes, the whole internal layer of the vein is degenerated with deformed, frag- mented structure [60]. Endothelium presents inflammatory profile and it is prone to adhesion and migration of blood components which is proved e.g. by elevated surface expression of CD146 [15, 60-62]. Endothelial cells release multiple inflammatory mediators and growth factors like von Willebrand factor (vWF) [12], osteoprotegerin [62], smooth muscle proliferation inducing factors (PGF2 $\alpha-$ prostaglandin $\mathrm{F} 2 \alpha, \beta \mathrm{FGF}$ - fibroblast growth factor $\beta$ ) [63], adhesion molecules intercellular adhesion molecule 1 (ICAM-1), vascular cell adhesion molecule 1 (VCAM-1), platelet endothelial cell adhesion molecule (PECAM-1) [64]. PECAM-1 has an anti-inflammatory function but it also takes part in preservation of vessel wall continuity [62]. It has a substantial role in clot dissolving during acute episodes of deep vein thrombosis - its lowered expression correlates with longer existence of thrombus (less macrophage infiltration, decreased angiogenesis) and increased fibrosis and the soluble form (sPECAM-1) could be a useful prognostic marker of postthrombotic syndrome [65].

Elevated concentration of monocyte/macrophage attracting factors in blood of varicose veins is probably not associated with endothelial cells, because removal of the endothelium does not cause decrease in expression of mRNA of these chemokines (IL-8, MCP-1, IP-10, RANTES, MIP- $1 \alpha$ and MIP-1 $\beta$ ) [66]. Endothelium of incompetent veins is more resistant to starvation (culture without serum or cytokines/growth factors), with decreased migration of its cells [62].

Another important feature of incompetent veins is increased angiogenesis accompanied by increased vessel permeability. It is caused probably by leukocytes adhering to the capillary wall and obstructing the vessels, releasing proteolytic enzymes and toxic metabolites [67]. Loss of larger molecules like fibrinogen causes formation of so called "cuffs" around the vein. These "cuffs" are often present in varices but their postulated role in formation of ulcerations has not been proved [68].

Venous intima has been shown to include an important subendothelial layer of pericytes which, unlike endothelial cells, have a prothrombotic profile. These cells store the tissue factor and in contrast to endothelium they cannot activate protein $C[69,70]$. Therefore, once endothelial barrier is damaged, its anticoagulatory properties are lost and the procoagulatory pericyte layer is exposed.

\section{Cytokines in chronic venous disease}

Lately Tisato et al. published two studies on cytokine profile of incompetent veins. In a study of a small group of 11 patients, among 31 cytokines tested the following cytokines were found in larger amounts as compared to control: IL-8, TNF- $\alpha$, GM-CSF, IFN- $\alpha 2$, MIP-1 $\beta$, VEGF, EGF, Eotaxin, MCP-1, PDGF and RANTES [71]. In another experiment with 31 patients with incompetent veins, out of 27 cytokines and chemokines assessed in the super- 
natant from endothelial cells culture only PDGF-BB was significantly correlated to the reflux time in the incompetent vein [72]. Administering pharmacological inhibitors of main PDGF-BB synthesis paths with TNF- $\alpha$ as a stimulator, it was shown that NF- $\mathrm{KB}$ signalling path was the main contributor to the PDGF-BB synthesis (TNF- $\alpha$ receptor activation starts multiple signalling pathways. As a result of one of them, NF- $\mathrm{BB}$ is activated. This pathway is known to take part in TNF- $\alpha$ inflammatory actions) [72].

Studies on correlation between plasma VEGF levels and severity of the illness according to CEAP scale show that despite VEGF increase with exacerbating symptoms, a statistical significance is noted only in patients with healed ulcers [73]. Nevertheless, an interesting paper on VEGF was published by Hollingsworth [74]. He assumed that macroscopic changes in incompetent veins are not continuous, so a molecular defect requiring additional factors must take part in the pathogenesis. He studied VEGF because of its crucial role for continuity and reactivity of vessels. Incompetent vein fragments were compared to competent fragments of the same varicose veins and with specimens from healthy people. Of all studied genes $\left(\mathrm{VEGF}_{121}, \mathrm{VEGF}_{165}\right.$ and for VEGF receptors: KDR, flt-1 and s.flt-1) only s.flt-1 transcription was not increased in varices. Elevated transcription of the four mentioned genes was not dependent on distance from the sapheno-femoral junction and did not differ between competent and incompetent fragments of varicose veins save $\mathrm{VEGF}_{121}$, for which transcription was greater in incompetent vein fragments. While transcription of s.flt-1 was not increased in varices, it was the only one which increased distally from sapheno-femoral junction. This occurred only when the sapheno-femoral junction was incompetent.

Jezovnik and Poredos [75] are convinced that inflammation takes part in pathogenesis of idiopathic thrombophlebitis. They examined patients 2-4 days after the first episode of idiopathic thrombophlebitis and they proved that even in this stable stage of the illness, IL-8, IL-6 and C-reactive protein (CRP) are significantly elevated. Elevated levels of endothelial dysfunction markers (P selectin and vWF) were also found. In a rat model of venous hypertension in limbs (arterio-venous fistula) [76], higher levels of IL-8 and increase in antigen/receptor Duffy in the skin were confirmed. Duffy receptor binds multiple chemokines.

\section{Implication for prevention and treatment}

Considering limited possibilities of non-invasive treatment and prevention, analysis of new potential targets of pharmacotherapy is an important issue. Currently available drugs are supposed to attenuate inflammation, unfortunately their effectiveness is questionable.

Vitamin C is considered important for vessel protection because apart from its antioxidative function it also takes part in collagen synthesis [14]. Calcium dobesilate also has a proved antioxidative effect in vitro in varices [77]. In hypoxic conditions, escin prevents activation of neutrophils and endothelium as well as diminishes adhesion and activation of HL60 cells (human promyelocytic leukemia cell line which can differentiate into granulocyte-like cells or macrophage/monocyte cells) [78]. Moreover - also in hypoxic environment - escin stops the decrease in ATP and the increase in phospholipase A2 [79]. In normoxemic conditions, however, no effect of escin could be found and the drug did not play any role in production of free radicals activated by a soluble stimulant (fMLP) [79]. It seems important, considering doubts about the existence of hypoxemia in varices [48]. Studies on vasoactive factors in varices show that escin could be useful rather at an early stage of the illness because in more advanced stages the vein wall becomes insensitive for contracting effect of the drug [80].

Flavonoids are considered effective in protection of cells from active oxygen forms. Their antioxidative function is based on scavenging of free radicals and other transient oxidative oxygen forms. They are also supposed to stop neutrophil infiltration and free radical creation through preventing the activating cascade induced by hypoxia [14]. Micronized purified flavonoid fraction (MPFF) significantly reduces venous reflux in an animal model of venous hypertension, protecting the valves from narrowing and decreasing the number of lymphocytes $\mathrm{T}$ on the valves $[41,80]$. Flavonoids administered for 60 days seem to decrease the plasma concentrations of some endothelium activation markers (ICAM-and VCAM) [81].

Unfortunately, some studies did not confirm any important impact of flavonoids on inflammatory markers (CD11b, CD18, IL-6, IL-8, selectins) [82]. Thirty days of diosmin administration in patients with healed venous ulcers did not show any anti-inflammatory effect (monitored by levels of cytokines and soluble adhesive molecules), on the other hand, an increased expression of adhesive molecule CD 11 b on circulating granulocytes was shown. This effect is attributed by the authors to changed interactions between leukocytes and endothelium or the direct impact of the flavonoid on granulocytes [82].

$300 \mathrm{mg}$ of acetylsalicylic acid administered daily ameliorates the healing of venous ulcers but its administration for 15 days prior to surgical removal of varicose veins did not decrease expression of monocyte attracting chemokines in vitro [83]. This fact along with the short time of drug administration may be responsible for the lack of effect [83].

Another substance examined for its anti-inflammatory effects and possibility of therapeutic use in chronic venous insufficiency is a glycosaminoglycan - sulodexide (SDX) [84]. It causes a significant decrease in the release of almost all cytokines, chemokines and adhesive molecules from macrophages stimulated by lipopolysaccharide. In 
a rat model of venous hypertension (arterio-venous fistula) [85] sulodexide decreased the expression of angiopoetin-2, simultaneously promoting expression of angiopoetin-1. According to the authors this may indicate ability to reduce endothelial hypertrophy in veins with hypertension. Sulodexide has antithrombotic and profibrinolytic properties and it can restore the endothelial glycocalyx [86]. Importantly, glycocalyx includes glycosaminoglycans which are larger than most adhesion molecules and as long as they are intact, they prevent contact between e.g. ICAM-1 and leukocytes, thus reducing the inflammatory processes $[19,86]$.

In the aforementioned study by Tisato et al. [71] in the next stages of the study two substances were examined: $\alpha$-lipoic acid and Ginkgo biloba derivative (GinkgoSelect phytosome). They both decreased cytokine expression in proinflammatory environment created by TNF- $\alpha$ addition. $\alpha$-lipoic acid which acts through NF- $\mathrm{B}$ and p38/MAPK path was more effective in comparison with Ginkgo biloba (acting through Akt path).

In a study from Philadelphia [45], MJ33, an indirect inhibitor of NOX was placed in anti-PECAM-1 immunoliposomes (Ab-MJ33/IL). They bound specifically with endothelium and neutralized angiotensin induced ROS production in vitro and in vivo. Moreover, AB-MJ33/IL turned out to be inhibitors of inflammatory marker VCAM expression in endothelium subject to TNF; they also diminished the damage in endothelial barrier caused by exposure to VEGF.

Summarizing, it can be stated that the inflammatory process is a significant part of venous insufficiency pathogenesis. Despite numerous studies there is still no clarity concerning the role of immunological cells in the aetiology of this illness, there are also few works on cytokine profile in varices. Such studies could help in targeting pharmacotherapy and as a result, better effectiveness of treatment and prophylaxis could be achieved.

\section{The authors declare no conflict of interest.}

\section{References}

1. Robertson L, Evans C, Fowkes FG (2008): Epidemiology of chronic venous disease. Phlebology 23: 103-111.

2. Evans CJ, Fowkes FG, Ruckley CV, Lee AJ (1999): Prevalence of varicose veins and chronic venous insufficiency in men and women in the general population: Edinburgh Vein Study. J Epidemiol Community Health 53: 149-153.

3. Rabe E, Pannier-Fischer F, Bromen K, et al. (2003): Bonner Venenstudie der Deutschen Gesellschaft für Phlebologie. Phlebologie 32: 1-14.

4. Carpentier PH, Maricq HR, Biro C, et al. (2004): Prevalence, risk factors, and clinical patterns of chronic venous disorders of lower limbs: a population-based study in France. J Vasc Surg 40: 650-659.
5. Zahariev T, Anastassov V, Girov K, et al. (2009): Prevalence of primary chronic venous disease: the Bulgarian experience. Int Angiol 28: 303-310.

6. Rabe E, Guex JJ, Puskas A, et al (2012): Epidemiology of chronic venous disorders in geographically diverse populations: results from the Vein Consult Program. Int Angiol 31: 105-115.

7. Jawień A, Grzela T (2004): Epidemiology of chronic venous disease. Przew Lek 8: 29-32.

8. Fiebig A, Krusche P, Wolf A, et al. (2010): Heritability of chronic venous disease. Hum Genet 127: 669-674.

9. Willenberg T, Schumacher A, Amann-Vesti B, et al. (2010): Impact of obesity on venous hemodynamics of the lower limbs. J Vasc Surg 52: 664-668.

10. Dindelli M, Parazzini F, Basellini A, et al. (1993): Risk factors for varicose disease before and during pregnancy. Angiology 44: 361-367.

11. Corcos L, De Anna D, Dini M, et al. (2000): Proximal long saphenous vein valves in primary venous insufficiency. J Mal Vasc 25: 27-36.

12. Lim CS, Davies AH (2009): Pathogenesis of primary varicose veins. Br J Surg 96: 1231-1242.

13. Raffetto JD, Khalil RA (2008): Mechanisms of varicose vein formation: valve dysfunction and wall dilation. Phlebology 23: 85-98

14. Glowinski J, Glowinski S (2002): Generation of reactive oxygen metabolites by the varicose vein wall. Eur J Vasc Endovasc Surg 23: 550-555.

15. Sayer GL, Smith PD (2004): Immunocytochemical characterisation of the inflammatory cell infiltrate of varicose veins. Eur J Vasc Endovasc Surg 28: 479-483.

16. Sprague AH, Khalil RA (2009): Inflammatory cytokines in vascular dysfunction and vascular disease. Biochem Pharmacol 78: 539-552.

17. Saharay M, Shields DA, Porter JB, et al (1997): Leukocyte activity in the microcirculation of the leg in patients with chronic venous disease. J Vasc Surg 26: 265-273.

18. Whiston RJ, Hallett MB, Davies EV, et al. (1994): Inappropriate neutrophil activation in venous disease. Br J Surg 81: 695-698.

19. Litwiniuk M, Grzela T, Brawura-Biskupski-Samaha R (2009): Chronic inflammation in venous leg ulcer - problems and perspectives. Centr Eur J Immunol 34: 247-251.

20. Ono T, Bergan JJ, Schmid-Schönbein GW, Takase S (1998): Monocyte infiltration into venous valves. J Vasc Surg 27: 158-166.

21. Knaapen MW, Somers P, Bortier H, et al. (2005): Smooth muscle cell hypertrophy in varicose veins is associated with expression of estrogen receptor-beta. J Vasc Res 42: 8-12.

22. Peyton BD, Rohrer MJ, Furman MI, et al. (1998): Patients with venous stasis ulceration have increased monocyte-platelet aggregation. J Vasc Surg 27: 1109-1115; discussion 11151106.

23. Rohrer MJ, Claytor RB, Garnette CS, et al. (2002): Platelet-monocyte aggregates in patients with chronic venous insufficiency remain elevated following correction of reflux. Cardiovasc Surg 10: 464-469.

24. Buján J, Pascual G, Bellón JM (2008): Interaction between ageing, inflammation process, and the occurence of varicose veins. Phlebolymphology 15: 123-130.

25. Hansson GK, Hermansson A (2011): The immune system in atherosclerosis. Nat Immunol 12: 204-212. 
26. Sharma AK, Lu G, Jester A, et al. (2012): Experimental abdominal aortic aneurysm formation is mediated by IL-17 and attenuated by mesenchymal stem cell treatment. Circulation 126 (Suppl 1): S38-45.

27. Ju X, Ijaz T, Sun H, et al. (2013): Interleukin-6-signal transducer and activator of transcription-3 signaling mediates aortic dissections induced by angiotensin II via the T-helper lymphocyte 17-interleukin 17 axis in C57BL/6 mice. Arterioscler Thromb Vasc Biol 33: 1612-1621.

28. Takase S, Schmid-Schönbein G, Bergan JJ (1999): Leukocyte activation in patients with venous insufficiency. J Vasc Surg 30: $148-156$.

29. Pappas PJ, Fallek SR, Garcia A, et al. (1995): Role of leukocyte activation in patients with venous stasis ulcers. J Surg Res 59: 553-559.

30. Konstantopoulos K, Neelamegham S, Burns AR, et al. (1998): Venous levels of shear support neutrophil-platelet adhesion and neutrophil aggregation in blood via P-selectin and beta2-integrin. Circulation 98: 873-882.

31. Smith PD (1999): Neutrophil activation and mediators of inflammation in chronic venous insufficiency. J Vasc Res 36 (Suppl 1): 24-36.

32. Hahn TL, Unthank JL, Lalka SG (1999): Increased hindlimb leukocyte concentration in a chronic rodent model of venous hypertension. J Surg Res 81: 38-41.

33. Summers C, Rankin SM, Condliffe AM, et al (2010): Neutrophil kinetics in health and disease. Trends Immunol 31: 318324.

34. Choi EY, Santoso S, Chavakis T (2009): Mechanisms of neutrophil transendothelial migration. Front Biosci (Landmark Ed) 14: $1596-1605$.

35. Kowalski J, Kośmider M, Pawlicki L, et al. (1997): Complement activates neutrophils during PTCA procedure in patients with unstable angina pectoris. Int J Cardiol 58: 229-240.

36. Mazzone A, Ricevuti G (1995): Leukocyte CD11/CD18 integrins: biological and clinical relevance. Haematologica 80 : 161-175.

37. Golias C, Tsoutsi E, Matziridis A, et al. (2007): Review. Leukocyte and endothelial cell adhesion molecules in inflammation focusing on inflammatory heart disease. In Vivo 21: 757-769.

38. Michiels C, Arnould T, Remacle J (2000): Endothelial cell responses to hypoxia: initiation of a cascade of cellular interactions. Biochim Biophys Acta 1497: 1-10.

39. Zeman K, Kantorski J, Paleolog EM, et al. (1996): The role of receptors for tumour necrosis factor-alpha in the induction of human polymorphonuclear neutrophil chemiluminescence. Immunol Lett 53: 45-50.

40. Michiels C, Arnould T, Thibaut-Vercruyssen R, et al. (1997): Perfused human saphenous veins for the study of the origin of varicose veins: role of the endothelium and of hypoxia. Int Angiol 16: 134-141.

41. Bergan JJ, Pascarella L, Schmid-Schönbein GW (2008): Pathogenesis of primary chronic venous disease: Insights from animal models of venous hypertension. J Vasc Surg 47: 183-192.

42. Wlaschek M, Scharffetter-Kochanek K (2005): Oxidative stress in chronic venous leg ulcers. Wound Repair Regen 13: 452461.

43. Guzik B, Chwała M, Matusik P, et al. (2011): Mechanisms of increased vascular superoxide production in human varicose veins. Pol Arch Med Wewn 121: 279-286.

44. Condezo-Hoyos L, Rubio M, Arribas SM, et al. (2013): A plasma oxidative stress global index in early stages of chronic venous insufficiency. J Vasc Surg 57: 205-213.
45. Hood ED, Greineder CF, Dodia C, et al. (2012): Antioxidant protection by PECAM-targeted delivery of a novel NADPH-oxidase inhibitor to the endothelium in vitro and in vivo. J Control Release 163: 161-169.

46. Krzyściak W, Kózka M (2011): Generation of reactive oxygen species by a sufficient, insufficient and varicose vein wall. Acta Biochim Pol 58: 89-94.

47. Krzyściak W, Kowalska J, Kózka M, et al. (2012): Iron content (PIXE) in competent and incompetent veins is related to the vein wall morphology and tissue antioxidant enzymes. Bioelectrochemistry 87: 114-123.

48. Lim CS, Gohel MS, Shepherd AC, et al. (2011): Venous hypoxia: a poorly studied etiological factor of varicose veins. J Vasc Res 48: 185-194.

49. Jacob MP, Cazaubon M, Scemama A, et al. (2002): Plasma matrix metalloproteinase- 9 as a marker of blood stasis in varicose veins. Circulation 106: 535-538.

50. Xu HM, Zhao Y, Zhang XM, et al. (2011): Polymorphisms in MMP-9 and TIMP-2 in Chinese patients with varicose veins. J Surg Res 168: e143-148.

51. de la Rosa G, Yang D, Tewary P, et al. (2008): Lactoferrin acts as an alarmin to promote the recruitment and activation of APCs and antigen-specific immune responses. J Immunol 180: 6868-6876.

52. Shields DA1, Andaz SK, Sarin S, et al. (1994): Plasma elastase in venous disease. Br J Surg 81: 1496-1499.

53. Serra R, Buffone G, Falcone D, et al. (2013): Chronic venous leg ulcers are associated with high levels of metalloproteinases-9 and neutrophil gelatinase-associated lipocalin. Wound Repair Regen 21: 395-401.

54. Grzela T, Niderla-Bielinska J, Litwiniuk M, White R (2014): The direct inhibition of MMP-2 and MMP-9 by an enzyme alginogel: A possible mechanism of healing support for venous leg ulcers. J Wound Care 23: 278-285.

55. Gomez I, Benyahia C, Louedec L, et al. (2014): Decreased PGE2 content reduces MMP-1 activity and consequently increases collagen density in human varicose vein. PLoS One 9: e88021.

56. Sansilvestri-Morel P, Fioretti F, Rupin A, et al. (2007): Comparison of extracellular matrix in skin and saphenous veins from patients with varicose veins: does the skin reflect venous matrix changes? Clin Sci 112: 229-239.

57. Shoab SS, Scurr JH, Coleridge-Smith PD (1998): Increased plasma vascular endothelial growth factor among patients with chronic venous disease. J Vasc Surg 28: 535-540.

58. Howlader MH, Smith PD (2003): Symptoms of chronic venous disease and association with systemic inflammatory markers. J Vasc Surg 38: 950-954.

59. Takase S, Pascarella L, Lerond L (2004): Venous hypertension, inflammation and valve remodeling. Eur J Vasc Endovasc Surg 28: 484-493.

60. Elsharawy MA, Naim MM, Abdelmaguid EM, Al-Mulhim AA (2007): Role of saphenous vein wall in the pathogenesis of primary varicose veins. Interact Cardiovasc Thorac Surg 6: 219-224.

61. Nüllen H, Noppeney T (2010): Diagnosis and treatment of varicose veins. Part 1: definition, epidemiology, etiology, classification, clinical aspects, diagnostic and indications. Chirurg 81: 1035-1044.

62. Tisato V, Zauli G, Voltan R, et al. (2012): Endothelial cells obtained from patients affected by chronic venous disease exhibit a pro-inflammatory phenotype. PLoS One 7: e39543. 
63. Schuller-Petrovic S, Siedler S, Kern T, et al. (1997): Imbalance between the endothelial cell-derived contracting factors prostacyclin and angiotensin II and nitric oxide/cyclic GMP in human primary varicosis. Br J Pharmacol 122: 772-778.

64. Khaleel MS, Dorheim TA, Duryee MJ, et al. (2012): High-pressure distention of the saphenous vein during preparation results in increased markers of inflammation: a potential mechanism for graft failure. Ann Thorac Surg 93: 552558.

65. Kellermair J, Redwan B, Alias S, et al. (2013): Platelet endothelial cell adhesion molecule 1 deficiency misguides venous thrombus resolution. Blood 122: 3376-3384.

66. Solá Ldel R, Aceves M, Dueńas AI, et al. (2009): Varicose veins show enhanced chemokine expression. Eur J Vasc Endovasc Surg 38: 635-641.

67. Agu O, Hamilton G, Baker DM, Dashwood MR (2002): Endothelin receptors in the aetiology and pathophysiology of varicose veins. Eur J Vasc Endovasc Surg 23: 165-171.

68. Falanga V, Kirsner R, Katz MH, et al. (1992): Pericapillary fibrin cuffs in venous ulceration. Persistence with treatment and during ulcer healing. J Dermatol Surg Oncol 18: 409-414.

69. Juchem G, Weiss DR, Gansera B, et al. (2010): Pericytes in the macrovascular intima: possible physiological and pathogenetic impact. Am J Physiol Heart Circ Physiol 298: H754-H770.

70. Nees S, Weiss DR, Juchem G, et al. (2013): Critical role of the vasa venarum in the pathogenesis of chronic venous disease. Part I: malperfused venules as pathogenetic hot spots. Phlebolymphology 20: 193-201.

71. Tisato V, Zauli G, Rimondi E, et al. (2013): Inhibitory effect of natural anti-inflammatory compounds on cytokines released by chronic venous disease patient-derived endothelial cells. Mediators Inflamm 2013: 423407.

72. Tisato V, Zamboni P, Menegatti E, et al. (2013): Endothelial PDGF-BB produced ex vivo correlates with relevant hemodynamic parameters in patients affected by chronic venous disease. Cytokine 63: 92-96.

73. Howlader MH, Coleridge Smith PD (2004): Relationship of plasma vascular endothelial growth factor to CEAP clinical stage and symptoms in patients with chronic venous disease. Eur J Vasc Endovasc Surg 27: 89-93.

74. Hollingsworth SJ, Powell Gl, Barker SG, Cooper DG (2004): Primary varicose veins: altered transcription of VEGF and its receptors (KDR, flt-1, soluble flt-1) with sapheno-femoral junction incompetence. Eur J Vasc Endovasc Surg 27: 259268.

75. Jezovnik MK, Poredos P (2010): Idiopathic venous thrombosis is related to systemic inflammatory response and to increased levels of circulating markers of endothelial dysfunction. Int Angiol 29: 226-231.

76. Huang W, Qin W, Lv L, et al. (2014): Duffy antigen / receptor for chemokines correlates with inflammatory reaction in rats with venous hypertension: implication for the pathogenesis of primary chronic venous disease. Vasa 43: 47-54.

77. Alda O, Valero MS, Pereboom D, et al. (2011): In vitro effect of calcium dobesilate on oxidative/inflammatory stress in human varicose veins. Phlebology 26: 332-337.

78. Bougelet C, Roland IH, Ninane N (1998): Effect of aescine on hypoxia-induced neutrophil adherence to umbilical vein endothelium. Eur J Pharmacol 345: 89-95.

79. Arnould T, Janssens D, Michiels C, Remacle J (1996): Effect of aescine on hypoxia-induced activation of human endothelial cells. Eur J Pharmacol 315: 227-233.
80. Brunner F, Hoffmann C, Schuller-Petrovic S (2001): Responsiveness of human varicose saphenous veins to vasoactive agents. Br J Clin Pharmacol 51: 219-224.

81. Shoab SS, Porter J, Scurr JH, Coleridge-Smith PD (1999): Endothelial activation response to oral micronised flavonoid therapy in patients with chronic venous disease - a prospective study. Eur J Vasc Endovasc Surg 17: 313-318.

82. Danielsson G, Norgren L, Truedsson L, et al. (2003): Flavonoid treatment in patients with healed venous ulcer: flow cytometry analysis suggests increased CD11b expression on neutrophil granulocytes in the circulation. Vasc Med 8: 83-88.

83. del Río Solá ML, Antonio J, Fajardo G, Vaquero Puerta C (2012): Influence of aspirin therapy in the ulcer associated with chronic venous insufficiency. Ann Vasc Surg 26: 620629.

84. Mannello F, Ligi D, Canale M, Raffetto JD (2014): Sulodexide down-regulates the release of cytokines, chemokines, and leukocyte colony stimulating factors from human macrophages: role of glycosaminoglycans in inflammatory pathways of chronic venous disease. Curr Vasc Pharmacol 12: 173-85.

85. Lei Y, Zheng Z, Wang Y, et al. (2013): Sulodexide may alleviate neointimal hyperplasia by inhibiting angiopoietin-2 in an arteriovenous fistula model. Mol Med Rep 7: 831-835.

86. Mannello F, Ligi D, Raffetto JD (2014): Glycosaminoglycan sulodexide modulates inflammatory pathways in chronic venous disease. Int Angiol 33: 236-242. 